\title{
PUMA - A New Channel Access Protocol for Wireless LANs
}

\author{
Marek Natkaniec \\ Department of Telecommunications \\ AGH University of Technology \\ Cracow, Poland \\ email: natkaniec@kt.agh.edu.pl
}

\author{
Andrzej R. Pach \\ Department of Telecommunications \\ AGH University of Technology \\ Cracow, Poland \\ email: pach@kt.agh.edu.pl
}

\begin{abstract}
In this paper we propose an extension of the IEEE 802.11 DCF protocol [5] called PUMA (Priority Unavoidable Multiple Access). PUMA can easy be implemented in wireless network cards instead of IEEE 802.11 DCF. It has been shown through simulation that PUMA is fair, efficient, stable and allows for provision of time-bounded services. Moreover, its performance is unaffected by hidden stations. The new backoff mechanism called DIDD (Double Increment Double Decrement) has been employed to increase the protocol efficiency especially in the case of heavy traffic load and large number of stations. The performance comparison between PUMA and IEEE 802.11 $D C F$ is presented. The obtained results show that PUMA behaves much better than IEEE 802.11 DCF.
\end{abstract}

Keywords

WLANs, MAC protocol, IEEE 802.11, DIDD backoff

\section{INTRODUCTION}

Wireless Local Area Networks (WLANs) are one of the most dynamically developing areas in communications. In addition to the mobility that becomes possible with WLANs, these systems can also be used in environments where the cable installation is expensive or impossible. They play a very important role in the network architecture as a provider of easy and unconstrained access to the wired infrastructure.

The detailed analysis of IEEE 802.11 can be found in [2], [3], [6], [8], [9]. It is clear the DCF function has many disadvantages. The main motivation of this paper is to design a new protocol by minor modifications of the existing IEEE 802.11 DCF function to eliminate these disadvantages. Since Ad Hoc networks employ the IEEE 802.11 DCF function, so only asynchronous traffic can be carried. The first introduced modification allows sending specified packets with priorities, what makes possible provision of isochronous services. The mechanism that allows for isochronousasynchronous traffic scaling can be introduced by employing additional timer T2. It plays the same role as the superframe in the PCF mode. The next modification, taken from the FAMA (Floor Acquisition Multiple Access) protocol, completely solves the hidden station problem through CTS over RTS packets domination in radio channel [4]. There are many protocols that utilize carrier sensing and RTS/CTS packet exchange but none of them can guarantee collision free data packet transmission. IEEE 802.11 DCF can only recognize the collision after the end of transmission (because of ACK packet lack) but it cannot prevent it. Some enhancements were introduced to increase the efficiency of operation, by adding a new backoff scheme called DIDD [7]. The packet-train mechanism was used to improve the protocol efficiency while sending very short data packets.

\section{DESCRIPTION OF PUMA}

The following modifications of the IEEE 802.11 DCF have been made to realize PUMA:

- domination of CTS over RTS in radio channel by extension of the CTS packet size,

- JAM signalling for realization of time-bounded services,

- additional T2 timer for isochronous-asynchronous traffic scaling,

- two modes of operation: RTS+CTS+DATA for asynchronous transmission and JAM+RTS+CTS+DATA for isochronous transmission,

- DIDD backoff mechanism,

- optional packet-train transmission mechanism,

- some changes in IEEE 802.11 control frames fields.

The PUMA protocol is intended for operation in ad-hoc networks. It can use, similarly to IEEE 802.11 , three physical layers: DSSS (Direct Sequence Spread Spectrum), FHSS (Frequency Hopping Spread Spectrum) and DFIR (Diffused Infra Red).

\section{Isochronous mode of transmission}

Time-bounded services can be implemented in PUMA. Since PUMA offers packet transmission with priority, it allows for isochronous service provision. It is possible with the aid of the special JAM signal that is transmitted in specified moments by all stations wishing to realize isochronous services.

Three different time intervals: SIFS, PIFS, DIFS (DIFS $>$ PIFS $>$ SIFS) are defined in PUMA protocol, similarly like in the IEEE 802.11 standard. Every station measures these time intervals after the end of data transmission to determine the moment they can start its own transmission. The station proceeds with its isochronous transmission if the medium is determined to be idle for an interval that exceeds the PIFS time. All stations sending isochronous packets should start its transmission in the same time and send the JAM signal. The JAM signal consists of pulses of energy and has the length of one slot. This signal informs all other stations (especially stations sending asynchronous 
packets) that for a moment in their neighbourhood, an isochronous transmission begins. It means that all other stations have to defer its transmission until reception of RTS or CTS packet to update their net allocation vector (NAV). The operation of JAM signal is similar to the busy tone in other multi-access protocols. A random interval (backoff interval) is then selected and is used to initialize the backoff timer. The backoff timer is decremented only when the medium is idle. It is frozen when the medium is busy until the next PIFS period. A station initiates a RTS packet transmission when the backoff timer reaches zero. This situation is presented in Figure 1.

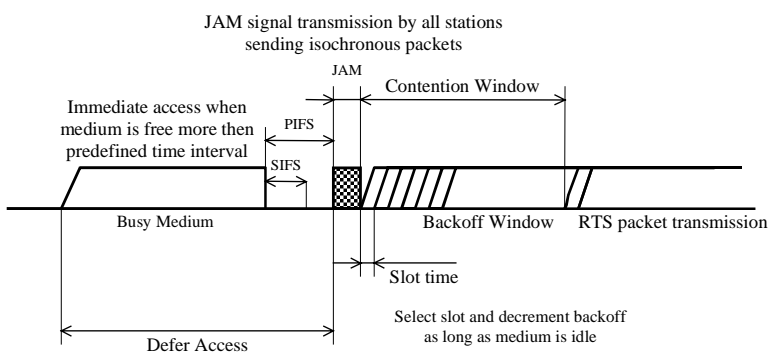

Figure 1. Channel access procedure for all stations sending isochronous packets.

On reception of a RTS packet the receiver responds with a CTS packet, which can be transmitted after the channel has been idle for a time interval exceeding SIFS. After the successful exchange of RTS and CTS packets the transmitter sends in collision free manner, the data packet after SIFS. In the case when a CTS packet is not received within the predetermined time interval, the RTS is retransmitted following the backoff rules. The PUMA protocol uses NAV - a timer that is always decreasing if its value is non-zero. A station is not allowed to initiate a transmission if its NAV is non-zero. The use of NAV to determine the busy/idle status of the channel is referred to as the Virtual Carrier sense mechanism. The typical isochronous packet transmission scenario is presented in Figure 2.

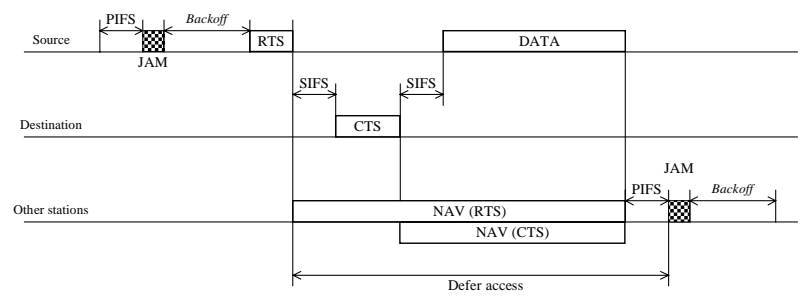

Figure 2. Isochronous packet transmission scenario in PUMA protocol.

It can be noted from the earlier study that transmission of small data packets is unprofitable because of large overhead [9]. Moreover, for each data transmission all contention procedures have to be run. The packet train mechanism has been implemented in PUMA to increase the protocol performance measures. Data packets are transmitted in sequence without collision after the successful medium reservation by a RTS/CTS packets exchange. The number of data packets transmitted in sequence can be set to the specific value. The idea of isochronous transmission enhanced by packet-train mechanism is presented in Figure 3.

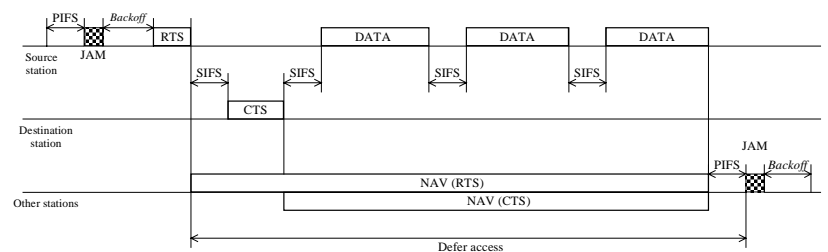

Figure 3. Typical isochronous transmission with packet-train mechanism enabled.

The idea of isochronous transmission assumes that packets generated by a traffic source should be delivered do its destination in the specified time. To accomplish this, the lifetime of each isochronous packet is measured. If it reaches its limit and the packet cannot be sent to its destination it is treated as useless and removed from the station buffer. The transmission procedure of an isochronous packet is presented in Figure 4.

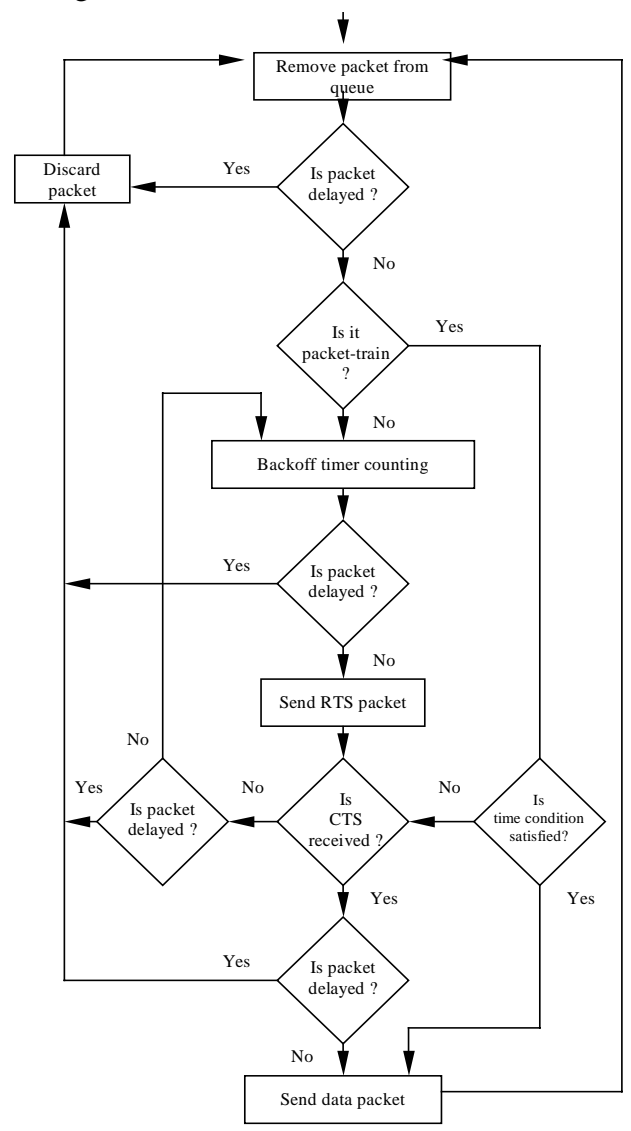

Figure 4. Transmission procedure of an isochronous packet.

\section{Asynchronous mode of operation}

This mode of operation is very similar to that of IEEE 802.11 DCF. The station proceeds with its asynchronous transmission if the medium is determined to be idle for an interval that exceeds the distributed interframe space 
(DIFS). In the case when the medium is busy the transmission is deferred until the end of ongoing transmission. A random interval (backoff interval) is then selected and the backoff timer is run. The backoff interval can be changed from the lower limit determined by the parameter CWmin to upper limit CWmax. In order to reduce the probability of collision, after each unsuccessful transmission attempt the expected value of the random backoff interval is increased exponentially up to the predetermined maximum. The backoff timer is only decremented when the medium is idle after DIFS time. It is frozen when the medium is busy. A station initiates a transmission of RTS packet when the backoff timer reaches zero.

In the case, when a CTS packet is not received within the SIFS time, it means that collision happened and the RTS is retransmitted following the backoff rules. The successful reception of CTS packet guarantees collision-free DATA packet transmission. Each station should update their NAV vector after receiving RTC or CTS.

The packet-train mechanism has been implemented in asynchronous transmission in the same manner like in the case of isochronous transmission. The data with additional CTS packets separated by SIFS intervals are transmitted in sequence without collision after the successful medium reservation by a RTS/CTS packets exchange. The only difference is that ACK packet finishes each transmission cycle.

\section{Isochronous-asynchronous traffic scaling}

The isochronous packets have a higher priority than asynchronous ones. It can happen that a large number of stations start to realize time-bounded services and then they kill asynchronous traffic (telnet, www, ftp, etc.). Such a situation is very undesirable. So, it is necessary to introduce a mechanism, which permits to control the minimal amount of asynchronous traffic (in IEEE 802.11 this is guaranteed by the superframe but it can only be used in infrastructure networks). The idea is to use an additional timer, which is used to measure the life-time of asynchronous packets located in source station buffer. An asynchronous packet located in the head of queue in the buffer gets a higher priority if its lifetime is reached (its priority is equal to the priority of isochronous packets). This packet will be certainty sent in the near future. All other asynchronous packets located in the buffer have the same low priority and after reception of ACK the life-time is measured using timer T2 for the next asynchronous packet (always located on the top of the buffer).

PUMA permits to regulate the minimum level of realized asynchronous traffic for every station. This level, of course, depends on the number of contending stations sending isochronous traffic.

The additional rule has been introduced in PUMA protocol to preserve an asynchronous station sending packets with packet-train mechanism enabled after T2 timeout. The asynchronous station can send then only one asynchronous data packet. This rule is necessary to assure the proper QoS level for isochronous transmission.

The name of the protocol - Priority Unavoidable Multiple Access (PUMA) came into being from the idea of timer T2 usage. The station sending asynchronous packets independently of the isochronous traffic load, will get after certain time (determined by T2 timeout) the possibility of contention with stations sending isochronous packets.

Timeout $\mathrm{T} 2$ can be dependent on the number of contending stations. It allows make the isochronous throughput independent from a number of stations sending asynchronous packets. Timeout T2 can be calculated for every station using the following formula:

$$
T 2=T 2^{\prime} \cdot \bar{N}
$$

where

$T 2$ ' - default T2 timeout assumed for station sending asynchronous packets,

$\bar{N}$ - the mean number of stations sending asynchronous packets located in the neighborhood of considered station (this value can be related with the number of stations sending isochronous packets).

The only problem is to determine the number of stations in the neighborhood of each station. However, the solution seems to be very simple since each station is obliged to listen the RTS/CTS packets and these packets carry information about the source and destination addresses, so it can create dynamically updated list of known addresses. A station can estimate the number of stations in its neighborhood on the basis of registered addresses. Obviously, if a given address does not appear in RTS/CTS packets for a long time it should be removed from the list. The described method permits to assure the desirable level of isochronous throughput. The growth of number of stations sending asynchronous packets should not influence the isochronous throughput.

\section{SIMULATION RESULTS}

In order to investigate these phenomena intensive simulations were performed. The overall performance of the PUMA protocol serving both data and real-time traffic was studied. The results obtained for asynchronous transmission have been compared with the IEEE 802.11 DCF protocol. The obtained simulations allowed to determine the realized throughput and the mean packet delay as a function of offered load for both transmissions types (the plots of the mean packet delay are not presented in this paper due to space limitation). The packet arrivals occurred according to the Poisson point distribution for all asynchronous transmissions. The voice streams were transmitted to realize isochronous services. The ON-OFF Brady model was used for all voice sources [1]. The method of nearly independent subruns was used in simulations. Each simulation point was obtained by running ten subruns and averaging the results. The plots present the curves, where for each simulation point for $95 \%$ confidence 
intervals the error does not exceed $1 \%$. Several assumptions were made to reduce the complexity of the simulation model:

- The effects of propagation delay are neglected. The channel is error-free, that is each packet that is transmitted by the sender is correctly received by the receiver.

- There are no stations operating in the power-saving mode.

- The stations are able to hear each other.

- There is no interference from nearby basic service sets (BSSs).

The network was configured to 2 Mbps medium capacity but the obtained results could also be referred to $11 \mathrm{Mbps}$ (IEEE $802.11 b$ ) due to the fact of protocol scalability (the standard changes are related to the physical layer). Some selected simulation results are presented in Figures 5 - 9.

The simulation results for asynchronous data transmission are presented in Figure 5. The comparison between IEEE 802.11 DCF and PUMA protocol is done. The DATA+ACK basic mode of transmission was used for IEEE 802.11 DCF. The PUMA protocol used mode RTS+CTS+DATA+ACK (the only possible).

The presented plot shows that PUMA protocol is very stable even in the case of a large number of stations working under heavy load (the maxima of throughput can be observed for the IEEE 802.11 DCF). The best results are achieved for IR, while the worst for FHSS, independently on the investigated protocol. The minimization of the length of interframe spaces, slot times and the physical layer preamble has positive influence on the network efficiency. The throughput efficiency gain using PUMA instead of IEEE 802.11 DCF protocol can be even up to $300 \%$.

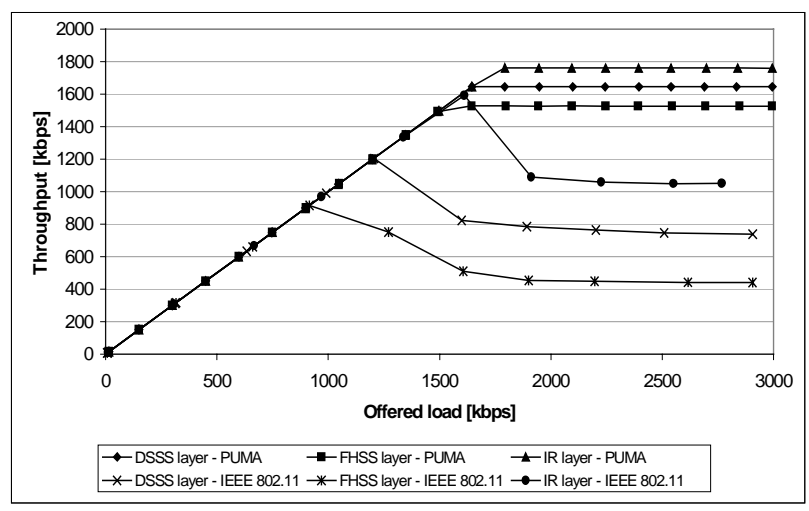

Figure 5. Asynchronous throughput as a function of offered load for 100 stations for DSSS, FHSS and IR.

The isochronous transmission results are presented in Figure 6. The ON-OFF source was assumed as a voice traffic model. The time spent in ON or OFF state is exponentially distributed with means of $1.0 \mathrm{~s}$ and $1.35 \mathrm{~s}$, respectively. The voice data is assumed to be encoded using the ITU G. 711 . The speech is encoded at $64 \mathrm{kbps}$ (for every $20 \mathrm{~ms}$ the 160 bytes packet is generated in the $\mathrm{ON}$ state). A packet is treated as useless and removed from the station buffer, if its lifetime has expired $(150 \mathrm{~ms})$. A few percent of isochronous packets loss was allowed. The numbers of implemented services with assumed QoS are as follows (all services are duplex ones): 24 for IR, 19 for DSSS and 16 for FHSS.

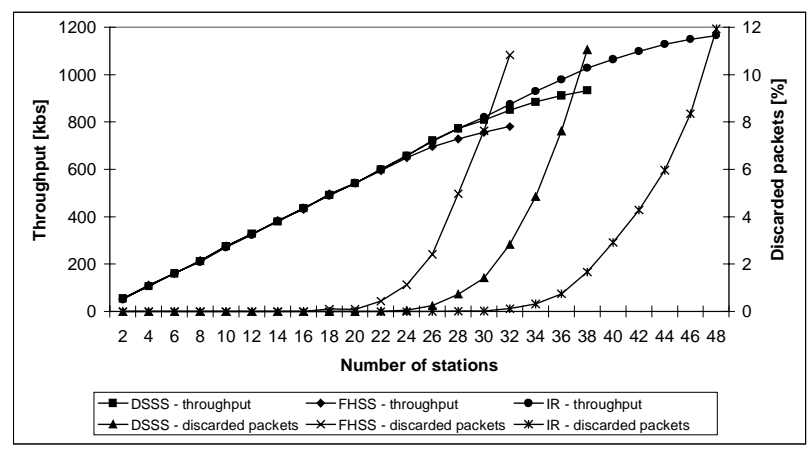

Figure 6. Throughput and percent of discarded packets versus number of stations (64 kbps voice streams).

The results of coexistence between isochronous and asynchronous transmissions are presented in Figures 7 - 9. Additional timer T2 allows for isochronous-asynchronous traffic scaling. Its usage allows for a change of the asynchronous packet priority. The ON-OFF source was again used to model the voice traffic. The different numbers of isochronous and fixed number of asynchronous stations were contended for channel access.

The isochronous throughput remains on the same level independently on asynchronous offered load. The PUMA protocol can make the most of available throughput by giving the remaining resources to the asynchronous stations. The asynchronous throughput increases up to the network limit (the end of free resources), then saturates on the constant level. The number of asynchronous stations has a marginal influence on asynchronous throughput. The PUMA protocol allows for a very efficient bandwidth use.

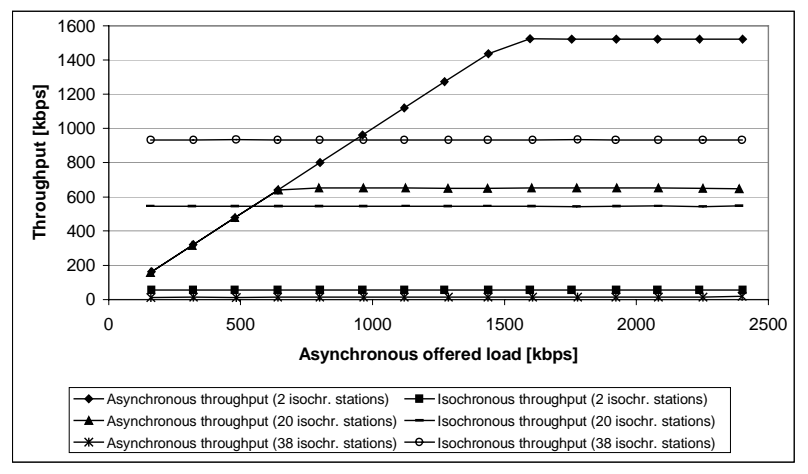

Figure 7. Isochronous and asynchronous throughput versus offered asynchronous load for 2, 20 and 38 isochronous (64 kbps voice streams transmission) and 2 asynchronous stations.

Timer T2 allows for controlling the asynchronous throughput level. Timer T2 related with the number of asynchronous stations has large influence on the isochronous throughput. Timer T2 changes have minor impact on the isochronous throughput for small number of isochronous services. We can observe a small degradation of isochro- 
nous throughput for lowest timer value, i.e. $5 \mathrm{~ms}$. More visible changes for a network heavily loaded with isochronous services can be observed. The asynchronous throughput replaces isochronous one for two smallest $\mathrm{T} 2$ timeouts. The most important conclusion is that the $\mathrm{T} 2$ timeout should be carefully selected otherwise the isochronous throughput can be seriously limited and percent of discarded isochronous packets can dangerously rise.

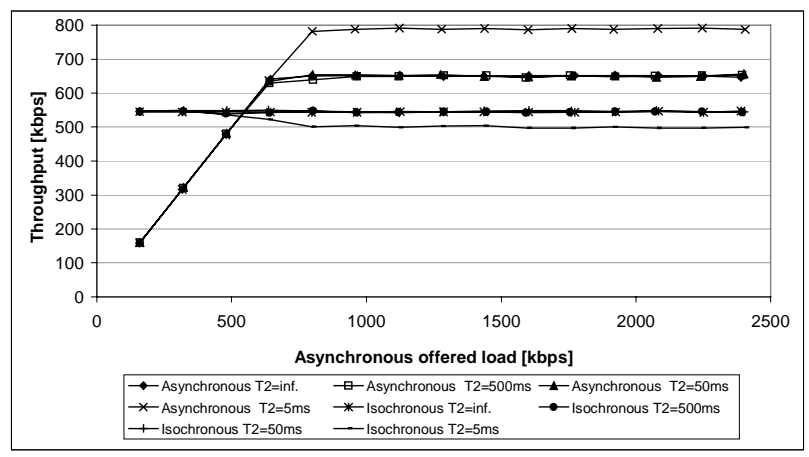

Figure 8. Isochronous and asynchronous throughput versus offered asynchronous load for 20 isochronous (64 kbps voice streams transmission) and 2 asynchronous stations for different T2 timeouts.

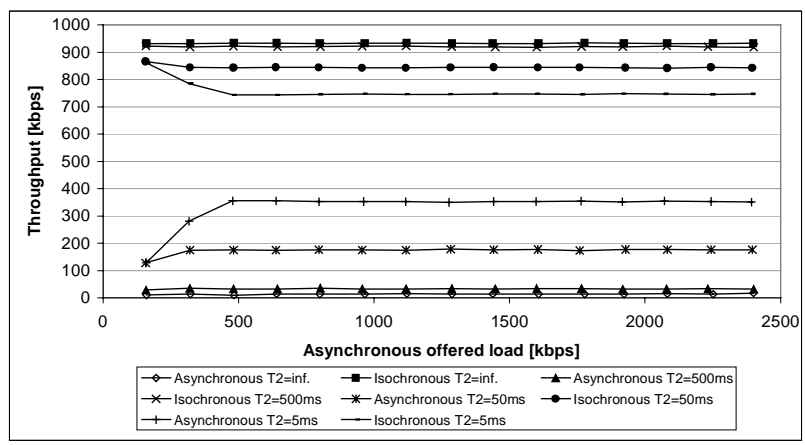

Figure 9. Isochronous and asynchronous throughput versus offered asynchronous load for 38 isochronous (64 kbps voice streams transmission) and 2 asynchronous stations for different T2 timeouts.

\section{CONCLUSIONS}

This paper presents a new medium access protocol called PUMA, which is a slight modification of IEEE 802.11 DCF. The simulation results show that the performance of PUMA is substantially increased in comparison with IEEE 802.11 DCF. The new DIDD backoff mechanism is especially designed for a network consisting of a large number of stations and working under heavy loads. It reduces the number of collisions, so brings the growth of the network efficiency. The idea of CTS over RTS packet domination in radio channel completely solves the hidden station problems. The packet-train mechanism allows to significantly increase the network efficiency especially in the case of very short data packets. The introduction of packet priorities (through JAM signaling) allows for realization time-bounded services. PUMA also permits to control the isochronousasynchronous throughput ratio (by maintaining additional timer). All the proposed enhancements could easy be adopted in the IEEE 802.11 DCF to increase the functionality and efficiency of the most popular WLAN protocol.

\section{REFERENCES}

[1] Brady P. T.: A Model for Generating On-Off Speech Patterns in Two-Way Conversation, Bell Systems Technical Journal, 48 (7), September 1969.

[2] Chhaya H. S., Gupta S.: Throughput and Fairness Properties of Asynchronous Data Transfer Methods in the IEEE 802.11 MAC Protocol, In Proc. PIMRC'95, September 27-29, Toronto, Canada, 1995.

[3] Crow B., Widjaja I., Kim G. J., Sakai P.T.: IEEE 802.11 Wireless Local Area Networks, IEEE Communications Magazine, September, 1997.

[4] Garcia-Luna Aceves J. J., Fullmer C., Floor Acquisition Multiple Access (FAMA) in Single-Channel Wireless Networks, ACM Mobile Networks and Applications Journal, Vol. 4, 1999.

[5] IEEE 802.11 Standard for Wireless LAN: Medium Access Control (MAC) and Physical Layer (PHY) Specification, New York, IEEE Inc., 1999.

[6] Natkaniec M., Pach A.R., An Analysis of Backoff mechanism in IEEE 802.11 standard., Proc. IEEE ISCC'2000, Antibes - Juans les Pins, France, 2000.

[7] Natkaniec M., Pach A.R., An Analysis of Modified Backoff Mechanism in IEEE 802.11 Networks, Proc. PGTS'2000, Dresden, Germany, 2000.

[8] Natkaniec M., Pach A.R.: An Analysis of the Influence of the RTS_Threshold Parameter on the IEEE 802.11 Network Performance, Proc. IEEE WCNC'2000 Wireless Communications and Networking Conference 2000, 2629 September, Chicago, U.S.A., 2000.

[9] Natkaniec M., Performance Analysis of Interconnections of IEEE 802.11 networks with other networks, Proc. WKTiI'99, Zegrze, Poland, 1999. 